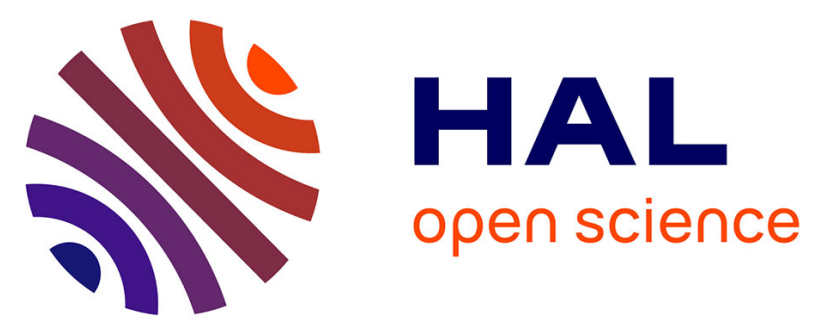

\title{
Cluster observations of finite amplitude Alfvén waves and small-scale magnetic filaments downstream of a quasi-perpendicular shock.
}

Olga Alexandrova, Anne Mangeney, M. Maksimovic, C. Lacombe, N. Cornilleau-Wehrlin, E.A. Lucek, Pierrette Décréau, J.-M. Bosqued, P. Travnicek, A.N. Fazakerley

\section{To cite this version:}

Olga Alexandrova, Anne Mangeney, M. Maksimovic, C. Lacombe, N. Cornilleau-Wehrlin, et al.. Cluster observations of finite amplitude Alfvén waves and small-scale magnetic filaments downstream of a quasi-perpendicular shock.. Journal of Geophysical Research Space Physics, 2004, 109 (A5), pp.A05207. 10.1029/2003JA010056 . hal-00153096

\section{HAL Id: hal-00153096 https://hal.science/hal-00153096}

Submitted on 17 Feb 2016

HAL is a multi-disciplinary open access archive for the deposit and dissemination of scientific research documents, whether they are published or not. The documents may come from teaching and research institutions in France or abroad, or from public or private research centers.
L'archive ouverte pluridisciplinaire HAL, est destinée au dépôt et à la diffusion de documents scientifiques de niveau recherche, publiés ou non, émanant des établissements d'enseignement et de recherche français ou étrangers, des laboratoires publics ou privés. 


\title{
Cluster observations of finite amplitude Alfvén waves and small-scale magnetic filaments downstream of a quasi-perpendicular shock
}

\author{
O. Alexandrova, ${ }^{1}$ A. Mangeney, ${ }^{1}$ M. Maksimovic,${ }^{1}$ C. Lacombe,${ }^{1}$ N. Cornilleau-Wehrlin, ${ }^{2}$ \\ E. A. Lucek, ${ }^{3}$ P. M. E. Décréau, ${ }^{4}$ J.-M. Bosqued, ${ }^{5}$ P. Travnicek, ${ }^{6}$ and A. N. Fazakerley ${ }^{7}$ \\ Received 23 May 2003; revised 28 August 2003; accepted 8 October 2003; published 11 May 2004.
}

[1] The Cluster satellites crossed the Earth's bow shock several times on 31 March 2001. For all these crossings the bow shock was supercritical and quasi-perpendicular. We present here the results of a detailed analysis of the magnetic field fluctuations observed downstream of the shock. We use data from the four Cluster spacecraft to determine the behavior and the geometry of these fluctuations with good accuracy. Shortly after the ramp crossing, we observed a large-amplitude nonlinear Alfvén wave, propagating along the downstream average magnetic field with a spectrum peaking at two frequencies below the proton and the alpha ion cyclotron frequencies. Farther downstream in the magnetosheath the magnetic field fluctuations took the form of three-dimensional structures which can be interpreted as cylindrical field-aligned current tubes. It is the first time that such current tubes have been observed downstream of a quasi-perpendicular shock, and they are closely associated with a quasi-monochromatic, finite amplitude Alfvén wave. We suggest that a close relation exists between the nonlinear Alfvén wave and the current tubes as a result of a filamentation instability which is expected to occur at $\beta \geq 1$ and for frequencies comparable to the ion cyclotron frequencies. INDEX TERMS: 2784 Magnetospheric Physics: Solar wind/magnetosphere interactions; 7851 Space Plasma Physics: Shock waves; 2728 Magnetospheric Physics: Magnetosheath; 2752 Magnetospheric Physics: MHD waves and instabilities; KEYWORDS: bow shock, Alfvén waves, current filaments

Citation: Alexandrova, O., A. Mangeney, M. Maksimovic, C. Lacombe, N. Cornilleau-Wehrlin, E. A. Lucek, P. M. E. Décréau, J.-M. Bosqued, P. Travnicek, and A. N. Fazakerley (2004), Cluster observations of finite amplitude Alfvén waves and small-scale magnetic filaments downstream of a quasi-perpendicular shock, J. Geophys. Res., 109, A05207, doi:10.1029/2003JA010056.

\section{Introduction}

[2] The structure of supercritical, quasi-perpendicular collisionless shocks is largely controlled by gyrating ions reflected at the shock ramp [see, e.g., Leroy et al., 1982; Paschmann et al., 1982]. Once they move downstream of the shock ramp, these ions contribute to the temperature anisotropy $T_{i \perp}>T_{i \|}$, where $T_{i \perp}$ and $T_{i \|}$ are the ion temperatures perpendicular and parallel, respectively, to an ambient magnetic field. This anisotropy is then the source of Alfvén ion cyclotron and/or mirror instabilities [see

\footnotetext{
${ }^{1}$ Observatoire de Paris-Meudon, Laboratoire d'Etudes Spatiales et d'Instrumentation en Astrophysique, CNRS, Meudon, France.

${ }^{2}$ Centre d'Etude des Environnements Terrestre de Planétaires, Institut Pierre Simon Laplace, Vélizy, France.

${ }^{3}$ Space and Atmospheric Physics, Imperial College, London, UK

${ }^{4}$ Laboratoire de Physique et Chimie de l'Environnement, CNRS, Orleans, France.

${ }^{5}$ Centre d'Etude Spatiale des Rayonnements, CNRS, Toulouse, France.

${ }^{6}$ Institute of Atmospheric Physics, Prague, Czech Republic.

${ }^{7}$ Mullard Space Science Laboratory, University College London, Dorking, UK.
}

Copyright 2004 by the American Geophysical Union. 0148-0227/04/2003JA010056
Schwartz et al., 1996]. When the proton thermal energy density is small with respect to the magnetic energy density, $\beta_{p}<1$, the most unstable mode is the Alfvén ion cyclotron mode (AIC), while the mirror mode is the most unstable one in the opposite situation. Furthermore, with a sufficient proportion of anisotropic $\alpha$ particles both the proton cyclotron and the helium cyclotron modes are destabilized [Gary et al., 1994].

[3] The nonlinear evolution of the AIC instability is not yet fully understood. Several saturation mechanisms may operate, either the quasi-linear relaxation [see, e.g., Yoon, 1992] or stronger nonlinearities like the parametric or modulational instabilities. In particular, if there is a perturbation with a finite component of the wave vector orthogonal to the ambient magnetic field $\mathbf{B}, k_{\perp}^{\prime} \neq 0$, the Alfvén wave may be unstable to a transverse instability, which generates field-aligned magnetic tubes, where the local concentration of the transverse magnetic energy increases. This phenomenon, called the filamentation instability, has been observed in Hall-MHD simulations for the parallelpropagating circularly polarized Alfvén wave pumps [Laveder et al., 2002].

[4] In this study we present Cluster observations suggesting that the filamentation instability may play a role 


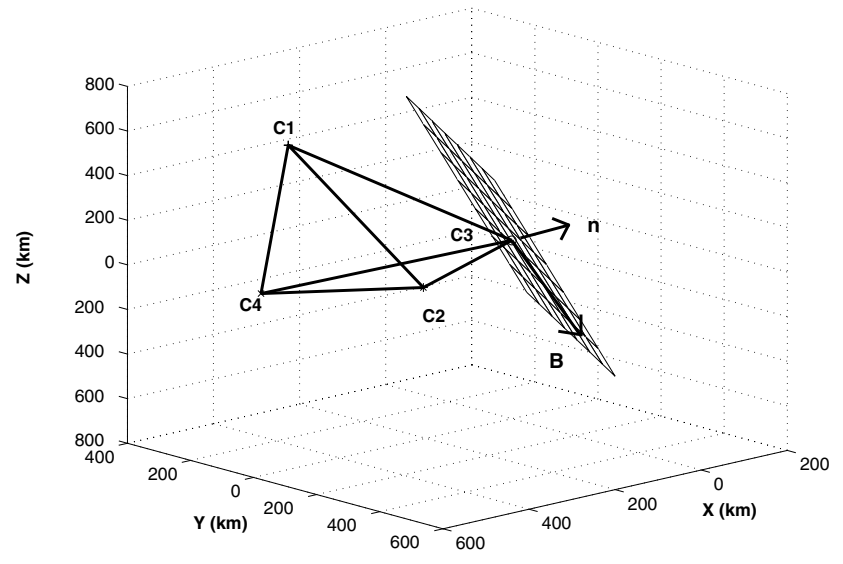

Figure 1. Relative positions of the four Cluster spacecraft $\left(C_{1}, C_{2}, C_{4}\right.$ to $\left.C_{3}\right)$ at the time of the shock crossing, the downstream magnetic field, and the plane of the shock front with its normal.

in the saturation of the AIC instability and in the temperature anisotropy relaxation downstream of quasiperpendicular shocks. These observations were obtained in the region immediately downstream of a supercritical, quasi-perpendicular bow shock where the plasma parameters were suitable for the development of the AIC instability: $\beta_{p}<1$ and simultaneously anisotropic protons and $\alpha$ particles. AIC waves were effectively observed downstream but close to the shock front with a relatively narrow spectrum centered on two frequencies comparable to the proton and $\alpha$ particle gyrofrequencies (see section 3); their steepened waveform displays evidence of significant nonlinearities.

[5] The most important result of this work is the identification, farther downstream, of field-aligned current tubes (section 4). Similar current tubes were already observed by the ISEE spacecraft [Rezeau et al., 1993] in the magnetopause boundary layer, while the present observations were made in the region downstream of the bow shock, just after crossing a region where a large-amplitude AIC wave was observed. We suggest that the current tubes, which look like the magnetic filaments (D. Laveder, private communication, 2003), result from the filamentation instability of the AIC wave.

\section{Observations}

[6] On 31 March 2001 the four Cluster spacecraft came out of the magnetosphere at $\sim 1440$ UT and crossed the bow shock for the first time at $\sim 1710 \mathrm{UT}$, the GSE coordinates of the magnetopause and bow shock crossings being (6.6, $0.2,8.8) R_{E}$ and $(9.4,-1.4,9.1) R_{E}$, respectively. After this crossing, nine successive shock crossings were observed until 2000 UT. All these shocks were quasi-perpendicular, the angle $\Theta_{B N}$ between the normal to the shock and the magnetic field ranging between $60^{\circ}$ and $90^{\circ}$ [Maksimovic et al., 2003].

[7] We have analyzed the magnetic field waveforms obtained by the search coils of the Spatio-Temporal Analysis of Field Fluctuations (STAFF) experiment [Cornilleau-Wehrlin et al., 1997, 2003] at a sampling frequency of $25 \mathrm{~Hz}$. Since these waveforms have been high-pass filtered to remove the spacecraft spin modulation, we have also used the data from the fluxgate magnetometer (FGM) experiment at 4-s resolution [Balogh et al., 2001] to recover the low frequencies. Both signals are combined by using a Haar wavelet transform (see Appendix A for more details) to retain the slow trends given by the smoothed FGM data as well as the fast variations of the STAFF magnetic waveforms. The "mixed" waveforms, which will be used throughout this study, are then obtained by inverting the resulting wavelet transform. We shall also use the potential of Haar wavelet transform to detect fast variations of the fields because of its good time resolution.

[8] In this paper, we shall examine in more detail an outward crossing at 1802:17 UT. A first estimate of the shock normal in the GSE system was obtained by using the time delays between the shock crossings by the four spacecraft [Schwartz, 1998; Maksimovic et al., 2003]. This yields a normal $\mathbf{n}_{*}=(0.94,-0.26,0.21)$ approximately parallel to the Sun-Earth direction. The relative positions of the four spacecraft at the time of the shock crossing, the downstream magnetic field, and the plane of the shock front with its normal are illustrated in Figure 1.

[9] The GSE direction of maximum magnetic field variance during the interval 1800-1804 UT around the shock crossing is $\mathbf{b}=(0.23,-0.13,-0.96)$; the direction varies very little between the four spacecraft. The maximum variance direction is expected to be orthogonal to the shock normal according to the coplanarity theorem. Thus we have chosen the unit vector parallel to $-\mathbf{b} \times$ $\left(\mathbf{b} \times \mathbf{n}_{*}\right)$ as the actual shock normal, $\mathbf{n}=(0.93,-0.26,0.26)$ $\simeq \mathbf{n}_{*}$. We shall use the direct shock coordinate system $(\mathbf{n}, \mathbf{b} \times \mathbf{n}, \mathbf{b})=(\mathbf{n}, \mathbf{m}, \ell)$ based on this "improved" shock normal.

[10] The magnetosheath and solar wind plasma parameters obtained by the Cluster $1\left(\mathrm{C}_{1}\right)$ and $\mathrm{ACE}$ spacecraft for this crossing are given in Table 1 . ACE data are taken with a 30-min delay corresponding to the solar wind travel time from the ACE location to the Earth orbit.

[11] The upstream and downstream average magnetic fields $\mathbf{B}_{s w}$ and $\mathbf{B}_{m s h}$ have been determined as averages over the two time intervals 1802:55-1804:07 UT upstream, and 1800:31-1801:43 UT downstream, respectively. They both have strong southward components in the GSE coordinate system; in the shock frame their components are $\mathbf{B}_{s w}=$ $(-2.4,3.4,29.5)$ and $\mathbf{B}_{m s h}=(-2.3,3.5,88.1) \mathrm{nT}$, which shows that the normal component of the magnetic field is approximately constant across the shock and that the coplanarity theorem applies reasonably well.

[12] The velocity data are obtained from the Cluster Ion Spectrometry (CIS) experiment [Rème et al., 1997] in the magnetosheath and from the ACE/Solar Wind Electron Proton Alpha Monitor [McComas et al., 1998] in the solar wind since CIS was in an operating mode that allowed good measurements only in the magnetosheath without ion separation. The bulk speeds are given in the rest frame of the shock, which moves with a local speed $\simeq 12 \mathrm{~km} / \mathrm{s}$ toward the Earth in the GSE system [Maksimovic et al., 2003]. The proton temperature $T_{p}$ is also taken from CIS downstream and from ACE upstream. The electron temperature $T_{e}$ is measured by 
Table 1. Magnetosheath and Solar Wind Plasma Parameters ${ }^{\mathrm{a}}$

\begin{tabular}{lcc}
\hline & Magnetosheath & Solar Wind \\
\hline$B, \mathrm{nT}$ & 90 & 30 \\
$f_{c p}, \mathrm{~Hz}$ & 1.4 & 0.5 \\
$f_{c c}, \mathrm{~Hz}$ & 0.7 & 0.2 \\
$V, \mathrm{~km} / \mathrm{s}$ & 260 & 603 \\
$T_{p}, \mathrm{eV}$ & 319 & 5 \\
$T_{e}, \mathrm{eV}$ & 87 & 39 \\
$N_{e}, \mathrm{~cm}^{-3}$ & 60 & 19 \\
$N_{p}, \mathrm{~cm}^{-3}$ & 49 & 16 \\
$N_{\alpha}, \mathrm{cm}^{-3}$ & 5.5 & 1.5 \\
$V_{A}, \mathrm{~km} / \mathrm{s}$ & 235 & 140 \\
$c / \omega_{p i}, \mathrm{~km}$ & 35 & 60 \\
$\beta_{p}$ & 0.8 & 0.04 \\
$\beta_{e}$ & 0.3 & 0.33 \\
\hline
\end{tabular}

${ }^{\mathrm{a}}$ Upstream velocity and $\alpha$ particle composition are observed by the ACE satellite; the other parameters are from the satellite Cluster 1. The bulk speeds are given in the rest frame of the shock.

the Plasma Electron and Current Experiment (PEACE) instrument [Johnstone et al., 1997].

[13] The electron concentration $N_{e}$ in the solar wind can be reliably estimated by the location of the plasma line by the Waves of High Frequency and Sounder for Probing of Elecron density by Relaxation (WHISPER) radio receiver [Décréau et al., 1997]. In the magnetosheath the plasma line, even if not easily distinguishable from the noise level, gives an electron density of around $60 \mathrm{~cm}^{-3}$. As noted above, the CIS instrument was in the magnetospheric mode for this particular shock crossing, and the CIS composition and distribution function (CODIF) sensor (with ion separation) was saturated because the proton concentration exceeded $5-10 \mathrm{~cm}^{-3}$. Therefore in the solar wind we use ACE data to determine the $\alpha$ particle abundance $N_{\alpha} / N_{p} \simeq 0.11$ and the quasi-neutrality to obtain the $\alpha$ and proton densities $N_{\alpha}$ and $N_{p}$. In the magnetosheath the CIS hot ion analyzer (HIA) sensor (without ion separation) gives a total ion concentration of $\sim 40 \mathrm{~cm}^{-3}$. This density does not satisfy a mass flux conservation across the bow shock; assuming a downstream abundance $N_{\alpha} / N_{p}$ equal to the upstream one, the mass flux conservation gives $N_{e}=56 \mathrm{~cm}^{-3}$ downstream. Therefore throughout this paper we will use the WHISPER result that is closer to this theoretical value and not the CIS density. Other parameters such as the Alfvén velocities $V_{A}=$ $B / \sqrt{\mu_{0} \rho}$ ( $\rho$ is the total mass density), the ion inertial lengths $c / \omega_{p i}$, and the proton and electron betas $\beta_{p}$ and $\beta_{e}$ are also presented in Table 1. In particular, the Alfvén Mach number of the shock is $M_{A}=V_{n} / V_{A}=4.4\left(V_{n}\right.$ is the upstream normal velocity in the shock rest frame), and the angle between the interplanetary magnetic field and the shock normal is $\Theta_{B N}=82^{\circ}$; therefore the shock is supercritical and quasi-perpendicular.

\section{The AIC Waves}

[14] Figure 2 displays the time profile of the three components of the magnetic field (in the shock frame) and of the proton temperature anisotropy $T_{p \perp} / T_{p \|}$ observed by the $\mathrm{C}_{1}$ spacecraft for a 12-min interval around the time of shock crossing at 1802:17 UT, taken as the origin of time for Figure 2 and most of the following analysis. An almost monochromatic wave can be observed in the $n$ and $m$ components of the magnetic field for $\sim 300 \mathrm{~s}$ downstream of the shock crossing. This is most clearly illustrated by using the Morlet wavelet transform (see Appendix A). The wavelet amplitudes $W B_{j}(f, t)$ give the contribution at time $t$ of fluctuations at scale $1 / f$ to the total fluctuation $\delta B_{j}(t)$ of the $j$ th component of the magnetic field. Figure 3 shows the contour levels in the timescale plane of the squared amplitude of the wavelet coefficients $\left|W B_{n}(f, t)\right|^{2}$ of the $n$ component of the magnetic field for the same time period as Figure 2 (here also $t=0$ at the shock crossing time). The scales $(1 / f)$ range from 0.1 to $18.0 \mathrm{~s}$, and the amplitude levels are represented by a color scale ranging from black (low) to red (high). The proton cyclotron period $1 / f_{c p}(\mathrm{~s})$ is shown by a solid line in Figure 3.

[15] One can see in Figure 3 the intense structured emission just before the shock crossing, corresponding to the quasi-sinusoidal wave seen in Figure 2. It lasts for $\sim 4 \mathrm{~min}$, from $280 \mathrm{~s}$ before the shock crossing to $30 \mathrm{~s}$ before the shock crossing, and peaks at 3-5 s scales. Deeper in the magnetosheath, at about $-350 \mathrm{~s}$, the fluctuation regime changes (see the vertical dashed line), and we observe intermittent peaks of energy, identified by black arrows, at scales between 1 and $2 \mathrm{~s}$, which will be discussed in section 4 .

[16] Simultaneous with the intense narrowband emission seen on the scalogram of Figure 3, a relatively large proton temperature anisotropy is observed (see the fourth panel of Figure 2). This anisotropy has a peak value just downstream of the shock ramp and reaches a more or less constant value of $\simeq 1.7$ deeper in the magnetosheath, when the field loses its quasi-sinusoidal character. It can therefore be expected that the observed wave is an AIC wave resulting from the development of an instability due to the particle anisotropies.

\subsection{Linear Theory}

[17] A linear analysis based on Vlasov theory, using an improved version of the linear dispersion code called WHAMP developed by Rönnmark [1982, 1983], confirms indeed that the level of anisotropy observed just downstream of the shock can be indicative of an AIC instability. For this, we have assumed bi-Maxwellian electron, proton, and $\alpha$ particle distribution functions, with parameters as close as possible to the observed values.

[18] In particular, the parallel electron and proton temperatures and the corresponding temperature anisotropies are taken as $T_{e \|}=85 \mathrm{eV}, T_{e \perp} / T_{e \|}=1.1$ (as observed by PEACE) and $T_{p \|} \simeq 170 \mathrm{eV}, T_{p \perp} / T_{p \|}=2.5$ (as observed by CIS), respectively. The $\alpha$ particle anisotropy is assumed to be the same as that of the protons, and the particle temperature is taken to be $T_{\alpha \|} \simeq 4 T_{p \|}$, a reasonable value downstream of a quasi-perpendicular bow shock with an upstream temperature ratio $\left(T_{\alpha \|} / T_{p \|}\right)_{s w}=4$ [Zhao et al., 1991; Fuselier et al., 1991]. The other parameters (magnetic field magnitude and concentrations) are taken from Table 1.

[19] With these parameters the most unstable modes are Alfvén waves propagating along the background magnetic field. The corresponding linear growth rate (Figure 4a) and frequency (Figure 4b) are displayed as functions of the normalized wave vector $k r_{p}$ parallel to the magnetic field, $r_{p}$ being the proton Larmor radius based on the 


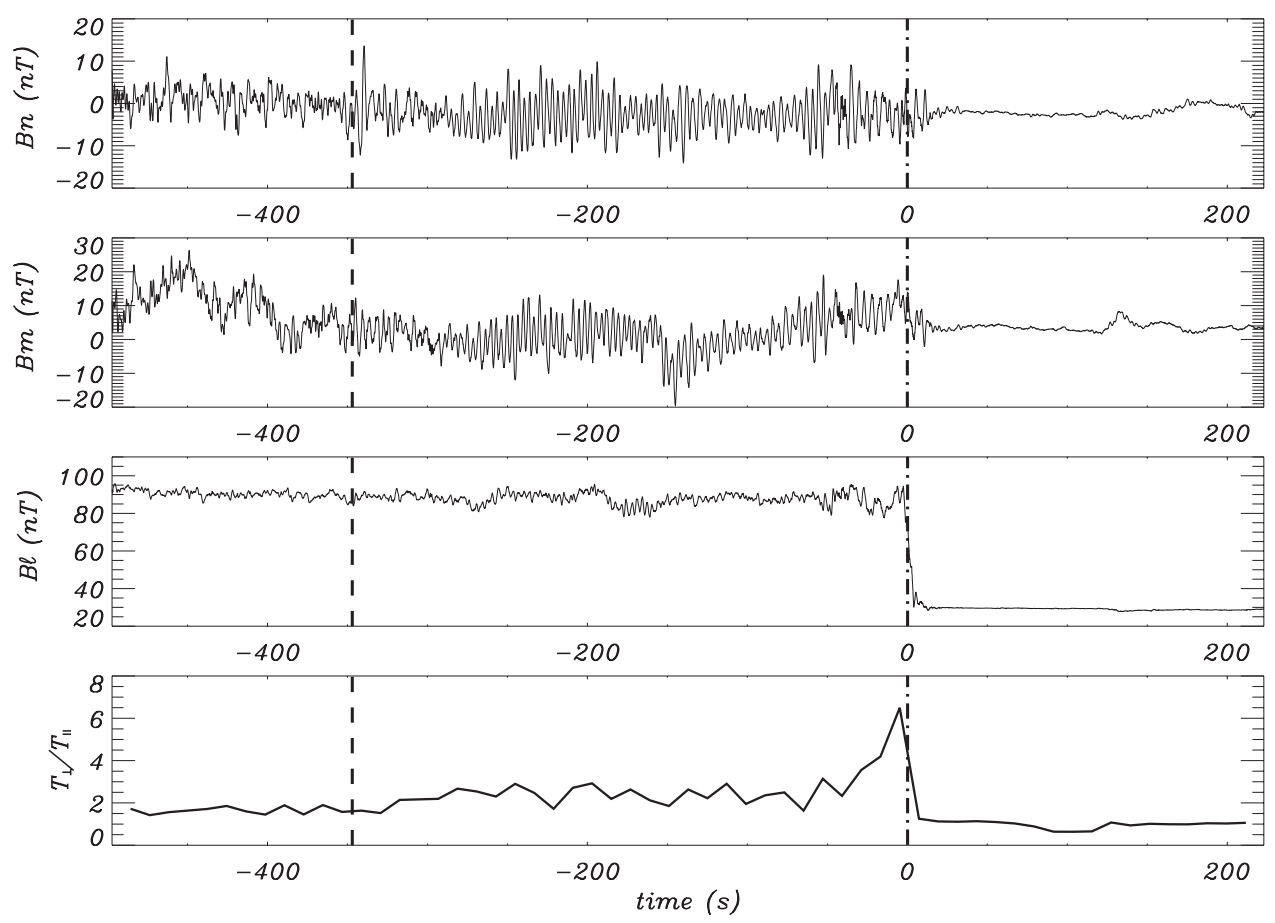

Figure 2. Cluster 1 magnetic field profiles (a) $\mathrm{B}_{n}$, (b) $\mathrm{B}_{m}$, (c) $\mathrm{B}_{l}$ in the shock frame and (d) the proton temperature anisotropy $T_{p \perp} / T_{p \|}$ for the interval 1754-1806 UT on $31 \mathrm{March} 2001$. The origin of times is taken at the shock crossing, 1802:17 UT, marked by the vertical dash-dotted line so that negative times correspond to the magnetosheath. The dashed vertical line corresponds to $t=1756: 30 \mathrm{UT}$ and separates the two fluctuation regimes discussed in text.

proton parallel thermal velocity $r_{p}=\left(2 k_{B} T_{p \|} / m_{p} f_{c p}^{2}\right)^{1 / 2} \simeq$ $23 \mathrm{~km}$.

[20] The growth rate is positive (instability) in two separate frequency domains centered on $0.24 f_{c p}$ and
$0.51 f_{c p}$, associated with the $\alpha$ particle and proton anisotropies, respectively. The corresponding unstable wave vector domains are centered on $k_{1} r_{p}=0.19$ and $k_{2} r_{p}=0.45$, respectively. The growth rate decreases with the angle

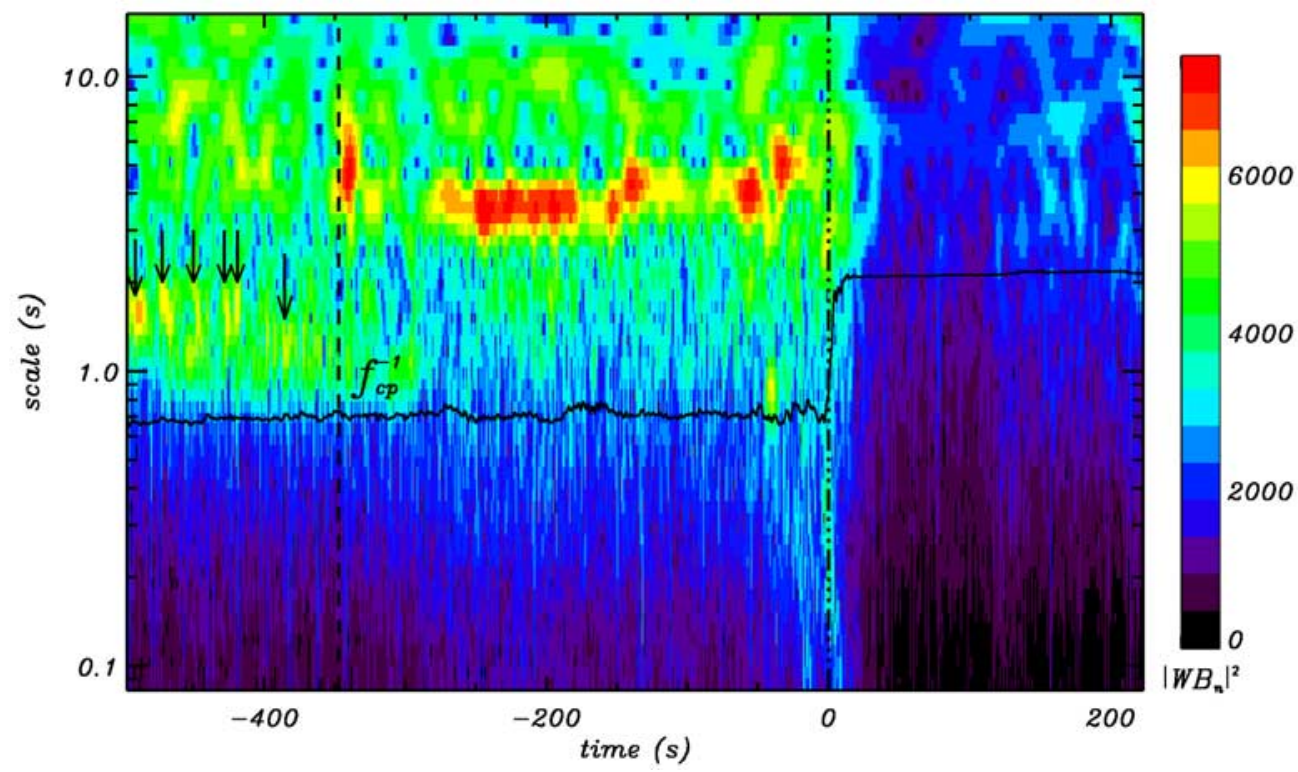

Figure 3. Dynamic spectrum of $B_{n}$, using the Morlet wavelet transform, around the bow shock crossing, 1754-1806 UT. The shock crossing time at 1802:17 UT is taken as the origin of time (vertical dashdotted line). The dashed vertical line corresponds to $t=1756: 30 \mathrm{UT}$; the proton cyclotron period, $1 / f_{c p}(\mathrm{~s})$, is shown by a black solid line; singular points (see section 4) are marked with black arrows. 

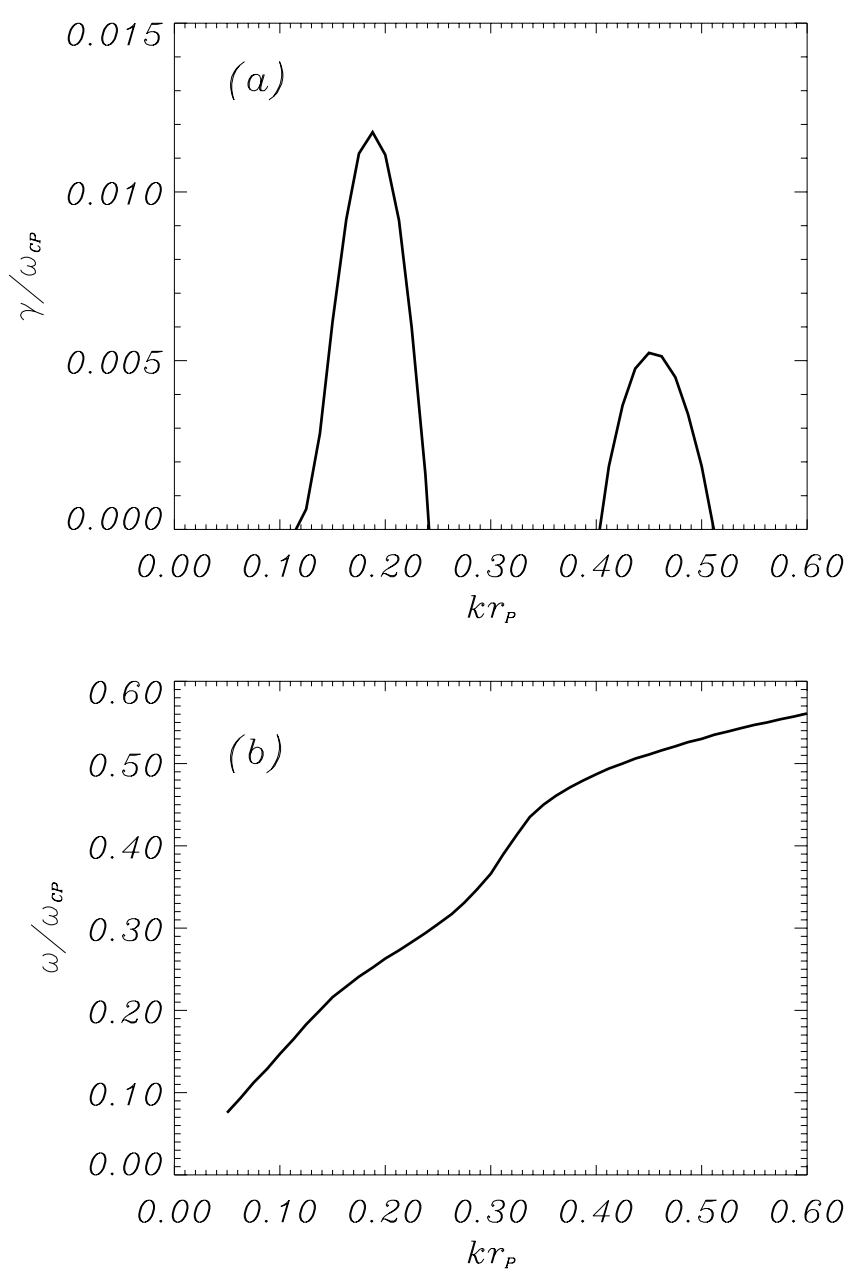

Figure 4. (a) Growth rate of the Alfvén ion cyclotron instability as a function of the nondimensional parallel wave vector $k r_{p}$. (b) Real part of the dispersion relation.

between the magnetic field and the wave propagation direction.

\subsection{Properties of the Narrowband Waves}

[21] We shall now show that the narrowband waves observed in the period 4 min prior to the shock crossing are indeed the expected AIC waves.

[22] First, a minimum variance analysis of the magnetic field [Sonnerup and Scheible, 1998] performed during a relatively uniform period from 260 to $170 \mathrm{~s}$ before the shock crossing for the satellite $C_{1}$ indicates that the direction of minimum variance, estimated to be parallel to the wave vector direction $\mathbf{e}_{\min }=(-0.19,0.07,0.98)$ in the shock (n, $\mathbf{m}, \mathbf{l})$ frame, is approximately parallel to the ambient magnetic field $\mathbf{B}_{m s h}$. The polarization of $\delta \mathbf{B}$ in the $(n, m)$ plane is circular and left-handed with respect to $\mathbf{B}_{m s h}$, as shown in Figure 5a. Therefore the polarization and propagation direction is compatible with that expected for an Alfvén wave propagating along the ambient magnetic field. The same results were obtained for the other three spacecraft.

[23] Second, a Fourier transform of the magnetic waveforms in the same uniform period (Figure $5 b$ ) shows that two frequencies are excited in the spacecraft frame: $f_{1}=0.29 \mathrm{~Hz}$ and $f_{2}=0.57 \mathrm{~Hz}$. There is approximately a factor of 2 between $f_{1}$ and $f_{2}$, indicating by comparison with Figure 4 that both the $\alpha$ particle and proton anisotropies contribute to the instability. Note that the spectral peak at the lower frequency $f_{1}$ is larger than the one at $f_{2}$, as predicted by the linear Vlasov theory, and dominates the waveform visible in Figure 2.

[24] In order to obtain the frequencies in the plasma rest frame, $f_{0,1}$ and $f_{0,2}$, the Doppler effect should be taken into account:

$$
\omega=\left|\omega_{0}+\left(\mathbf{k} \cdot \mathbf{V}_{b}\right)\right|,
$$

where $\omega=2 \pi f$ is the observed frequency in the satellite frame (assumed to be at rest in the GSE frame of reference), $\mathbf{V}_{b}=270(-0.95,0.11,0.29) \mathrm{km} / \mathrm{s}$ is the magnetosheath plasma bulk velocity in the GSE frame for the wave observation period, and $\mathbf{k}$ is the still unknown wave vector.

[25] The wave vector can be determined by using data from the four Cluster spacecraft [Balikhin et al., 1997, 2000; Sahraoui et al., 2003]. Indeed, let us make a Fourier transform of one component of the magnetic field, say, $B_{X}(t, i)$, where $i$ denotes the spacecraft. At a given frequency $f$ the Fourier amplitude $\hat{B}_{X}(f, i)$ is a complex number with a
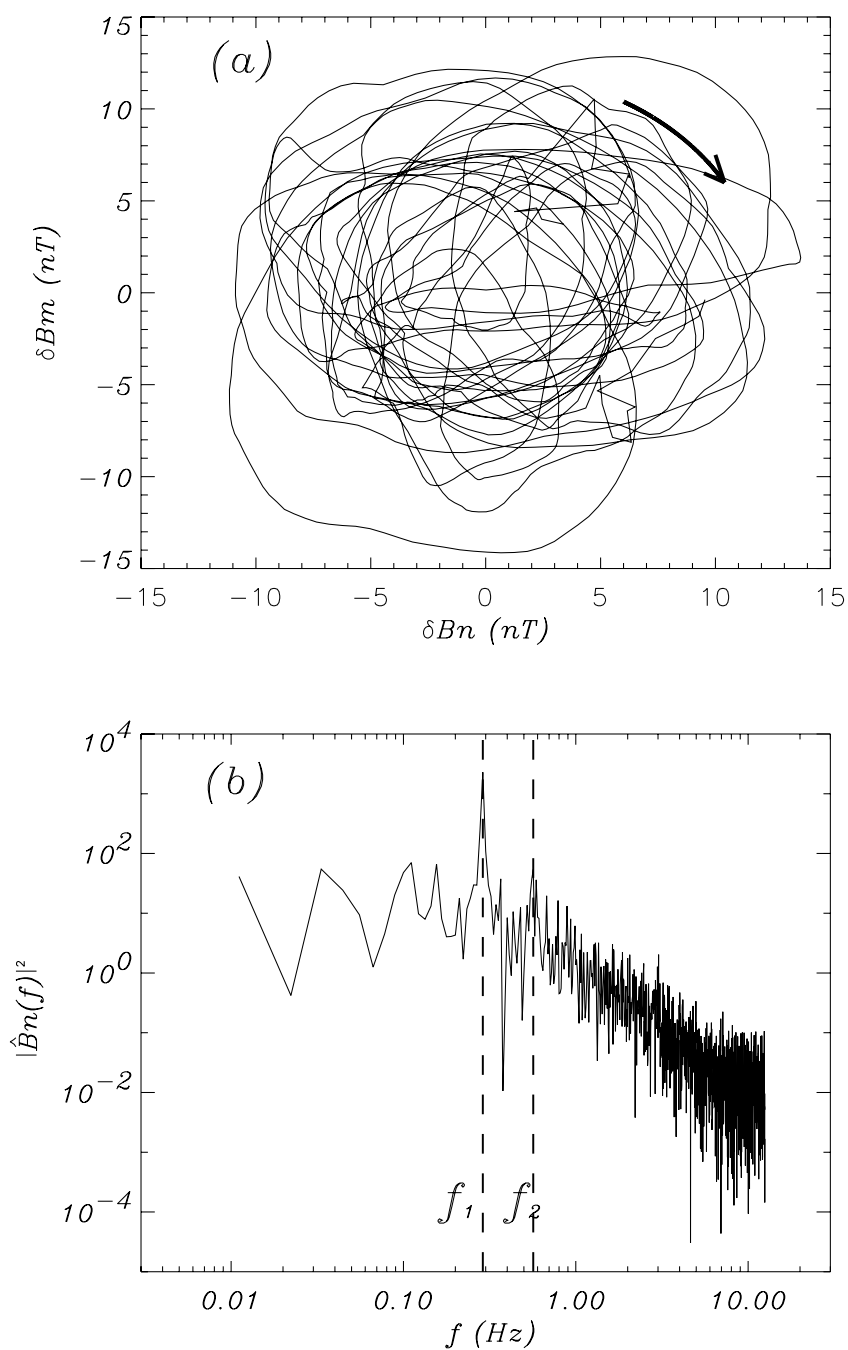

Figure 5. (a) Hodogram. (b) Fourier transform of $B_{n}$ over the period from 260 to $170 \mathrm{~s}$ before the shock crossing. The spectral density $\left|\hat{B}_{n}(f)\right|^{2}$ has two peaks over the frequencies $f_{1}=0.29$ and $f_{2}=0.57 \mathrm{~Hz}$ (dashed lines). 
phase $\varphi_{i}(f)$. Taking $\mathrm{C}_{1}$ as the reference spacecraft, the phase difference between the magnetic waveforms observed on $\mathrm{C}_{2}, \mathrm{C}_{3}$, and $\mathrm{C}_{4}$ and that observed on $\mathrm{C}_{1}$ can be written as

$$
\left[\varphi_{1}(f)-\varphi_{i}(f)\right]=\left(\mathbf{k} \cdot \delta \mathbf{r}_{1 i}\right)+2 \pi n_{1 i}, \quad i=2,3,4
$$

where $\delta \mathbf{r}_{1 i}=\mathbf{r}_{1}-\mathbf{r}_{i}$ is the separation vector between $\mathrm{C}_{1}$ and $\mathrm{C}_{i}$. This is a system of three equations for the three components of the wave vector, which depends on unknown parameter $n_{1 i}=0, \pm 1, \ldots$. This indeterminacy can be lifted by imposing some requirements: (1) The solution must be parallel to the direction of minimum variance $\mathbf{e}_{\min }$; (2) the angle between the magnetic field and $\mathbf{k}$ is expected to be small, as predicted by the linear theory; (3) the function $\sum_{i}\left\{\left[\varphi_{1}(f)-\varphi_{i}(f)\right]-\left(\mathbf{k} \cdot \delta \mathbf{r}_{1 i}\right)-2 \pi n_{1 i}\right\}^{2}$ must be minimal; and (4) we choose the smallest $n_{1 i}$ compatible with the first three requirements. In this way we obtain (in the GSE system of coordinates)

$$
\mathbf{k}_{1}=14 \times 10^{-3}(0.27,-0.26,-0.93) \mathrm{km}^{-1}
$$

for the wave observed at frequency $f_{1}=0.29 \mathrm{~Hz}$, and

$$
\mathbf{k}_{2}=25 \times 10^{-3}(-0.07,-0.23,-0.97) \mathrm{km}^{-1}
$$

for the wave observed at frequency $f_{2}=0.57 \mathrm{~Hz}$. In the (n, $\mathbf{m}, \ell$ ) frame the unit vectors of $\mathbf{k}_{1}$ and $\mathbf{k}_{2}$ are

$$
\mathbf{e}_{1 *}=(0.08,0.12,0.99) \quad \mathbf{e}_{2 *}=(-0.25,0.18,0.95) .
$$

These wave vectors are approximatively parallel to the ambient magnetic field: The angle between $\mathbf{e}_{1 *}$ and $\mathbf{B}_{m s h}$ is $\sim 10^{\circ}$; for the second mode this angle is $\sim 15^{\circ}$.

[26] Using equation (1), we can now determine the wave frequencies in the plasma rest frame:

$$
f_{0,1}=0.62 \mathrm{~Hz} \quad f_{0,2}=0.83 \mathrm{~Hz}
$$

and the corresponding parallel phase velocities $V_{\varphi \|}=\omega_{0} / k_{\|}$. For the first wave mode we find $V_{\varphi \|} \simeq 280 \mathrm{~km} / \mathrm{s}$; for the second mode we find $V_{\varphi \|} \simeq 215 \mathrm{~km} / \mathrm{s}$. These values are compatible, within the observational uncertainties, with the estimated Alfvén speed in the magnetosheath (see Table 1).

[27] Furthermore, there is a qualitative agreement with the predictions of the linear Vlasov theory. The two solutions we found, $k_{1 \|} r_{p}=0.31$ and $k_{2 \|} r_{p}=0.55, f_{0,1}=$ $0.43 f_{c p}$ and $f_{0,2}=0.58 f_{c p}$, are in a good agreement with the nonlinear dispersion relation presented in Figure $4 \mathrm{~b}$. However, they do not correspond exactly to the maxima of the growth rate. A better quantitative agreement could be achieved by changing the parameters used in the linear theory; however, as discussed in section 2, some of these parameters are so uncertain that the qualitative agreement achieved here is the only one which is meaningful.

[28] Therefore the polarization, the dispersive properties of the waves, and the comparison with the linear theory confirm the identification of the observed wave modes at $f_{1}$ and $f_{2}$ as Alfvén ion cyclotron waves excited by the anisotropy of the $\alpha$ particles and the protons, respectively.

[29] The waveforms of the AIC are periodic, but a detailed analysis reveals that they are not sinusoidal and have steepened wave fronts, indicating the presence of relatively strong nonlinearities. This is apparent in Figure 6, which

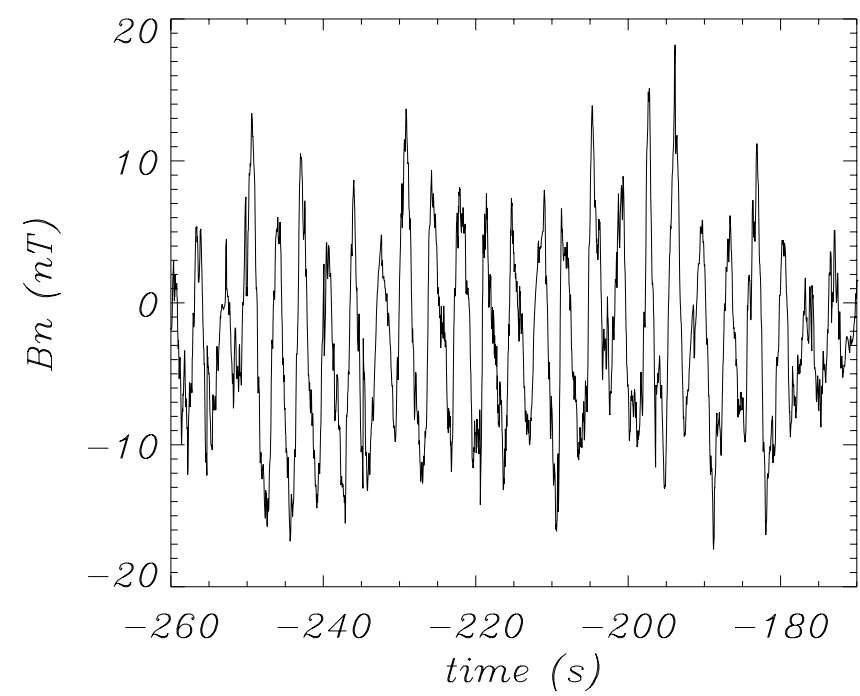

Figure 6. Close-up of Figure 2, component $B_{n}$ shown with the same origin of time.

displays the profile of the magnetic field component $B_{n}$ for the period from 260 to $170 \mathrm{~s}$ before the shock crossing.

[30] A more systematic analysis can be made by using the Haar wavelet transform (see Appendix A), which we shall apply to this period when the AIC waves have a relatively uniform behavior. A local gradient will be qualified as steep when the local energy at the smallest scale (i.e., the square of the wavelet coefficient calculated on $\Delta t=0.08 \mathrm{~s}$ ) exceeds by some threshold value $\eta>1$ the mean energy of the fluctuations at this scale: $\left|W B_{X}\right|^{2}>\eta\left\langle W B_{X}^{2}\right\rangle$, the average $\left\langle W B_{X}^{2}\right\rangle$ being taken on the whole interval of analysis. Note that the result of the analysis does not depend on the particular value of $\eta$ as soon as it is larger than 3-5.

[31] Then a minimum variance analysis of the magnetic field fluctuations over a time interval of $2 \mathrm{~s}$ is made around each time when a "steep" gradient is detected. The number of points with a steep gradient obviously depends on the value of $\eta$, but in most cases only one component of the magnetic field has a large variation, while the other two remain almost constant. The component of maximal variance is always orthogonal to the large-scale magnetic field $\mathbf{B}_{m s h}$. This is illustrated in Figure 7, which displays the profiles of the three components of $\delta \mathbf{B}$ in the local minimum variance frame for an interval of $\Delta t=2 \mathrm{~s}$ around the "singularity" (a time with a steep gradient) occurring at $t_{\text {sing }} \simeq-220 \mathrm{~s}(1758: 38 \mathrm{UT})$. Here the directions of maximum, intermediate, and minimum variance are $x^{\prime}, y^{\prime}$, and $z^{\prime}$, respectively. One can see that $\delta B_{x^{\prime}}$ changes sign at $t_{\text {sing }}$ and that the other two components fluctuate around zero. The eigenvalues of the variance tensor [Sonnerup and Scheible, 1998] are 1, 0.33, and 0.03, respectively, the direction of minimum variance making an angle of $\simeq 15^{\circ}$ with $\mathbf{B}_{m s h}$. Therefore these "singularities" are current sheets approximately parallel to the wave fronts, presumably produced by some nonlinear steepening of the AIC waves.

\section{Three-Dimensional Structures: Current Tubes}

[32] The scalogram of Figure 3 shows that the intense $\alpha$ particle AIC wave at scale $1 / f_{1} \simeq 3.6 \mathrm{~s}$ disappears abruptly 


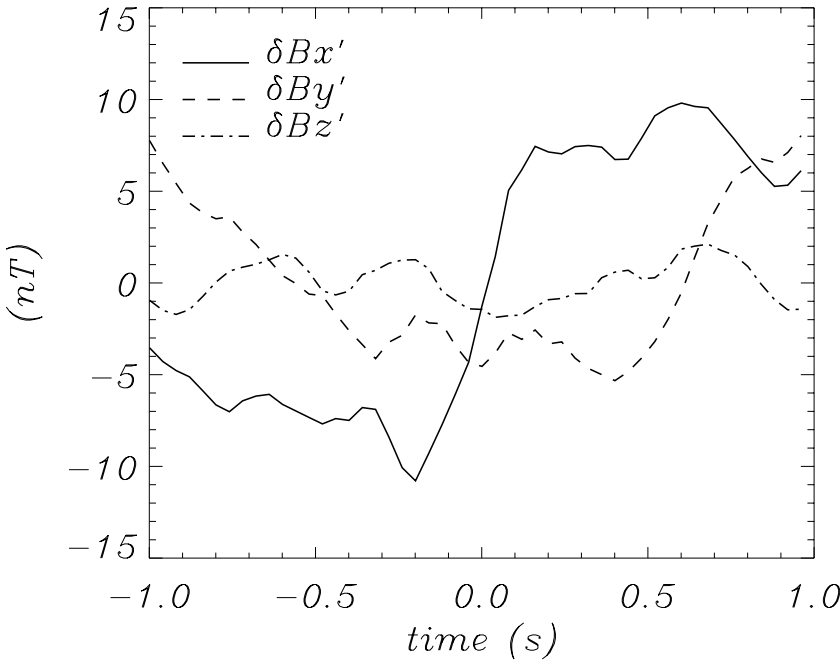

Figure 7. Profiles of the three components of $\delta \mathbf{B}$ in the minimum variance frame $\left(x^{\prime}, y^{\prime}, z^{\prime}\right)$ around the steep gradients at time $t_{\text {sing }} \simeq-220 \mathrm{~s}(1758: 38 \mathrm{UT})$ downstream of the shock front.

deep in the magnetosheath, i.e., before -350 s. However, during the time interval from 500 to $350 \mathrm{~s}$ before the shock crossing, the level of magnetic field turbulence remains relatively high, with strong localized peaks, marked by the arrows, at scales between 1 and $2 \mathrm{~s}$. One may consider these peaks as "singular" points, i.e., times at which a steep gradient is observed, as discussed in section 3.2. The time width of these localized peaks is significantly bigger than the sampling time, so to identify the singular points in this region, the time resolution of the Morlet wavelet transform (WT) is sufficient, and we do not need the high time resolution of the Haar WT (see Appendix A). Here also, the number of singular points depends on the threshold $\eta$, but above a minimal value they all share the same properties, which are significantly different from the planar current sheets discussed in section 3.2.

[33] Let us consider one of these singularities occurring at $\tau \simeq-420 \mathrm{~s}(1755: 16 \mathrm{UT})$. During a 10-s period around $\tau$, $\mathrm{C}_{1}$ observes a regular magnetic field profile as illustrated by the component $\delta B_{x^{\prime}}$ in Figure $8 \mathrm{a}$, where $x^{\prime}$ is the maximum variance direction in the local variance frame, calculated over the period under consideration. The variance analysis of $\delta \mathbf{B}$ for this period gives the three eigenvalues $1,0.68$, and 0.14 , so the singularity has a higher dimension than the current sheets. In Figure $8 \mathrm{~b}$ the three components $\left(\delta B_{x^{\prime}}\right.$, $\left.\delta B_{y^{\prime}}, \delta B_{z^{\prime}}\right)$ of $\delta \mathbf{B}$ in the same minimum variance frame are displayed for the 2-s interval around $\tau$ (the period between the two vertical dotted lines in Figure 8a). One can see that $\delta$ B has a three-dimensional (3-D) structure: None of the components remains constant, contrary to the case of the current sheets, even if the fluctuation in $\delta B_{z^{\prime}}$ is smaller than that of the two other components. The direction of minimum variance is approximately parallel to $\mathbf{B}_{m s h}$, indicating that the axis of the symmetry of the 3-D structure is field aligned.

[34] As seen in Figure 1, the separation vector (in GSE) $\delta \mathbf{r}_{14}=(46.0,-23.6,654.3) \mathrm{km}$ between $\mathrm{C}_{1}$ and $\mathrm{C}_{4}$ is almost parallel to $\mathbf{B}_{m s h}$. The angle between these two vectors is $19^{\circ}$.
If the structures observed by $\mathrm{C}_{1}$ are field aligned, it is expected that they will be found in the $\mathrm{C}_{4}$ data. Indeed, a good correlation between the field variations observed on $\mathrm{C}_{1}$ and $\mathrm{C}_{4}$ are found for the singularities occurring around $t_{\text {sing }}^{\mathrm{cl}}=1755: 10$ and $t_{\text {sing }}^{\mathrm{c} 4}=1755: 11$, respectively:

$$
\begin{aligned}
& C\left(\delta B_{x^{\prime}}^{\mathrm{cl}}, \delta B_{x^{\prime}}^{\mathrm{c} 4}\right)=0.98, \\
& C\left(\delta B_{y^{\prime}}^{\mathrm{c} 1}, \delta B_{y^{\prime}}^{\mathrm{c} 4}\right)=0.78, \\
& C\left(\delta B_{z^{\prime}}^{\mathrm{c} 1}, \delta B_{z^{\prime}}^{\mathrm{c} 4}\right)=0.27
\end{aligned}
$$

Figure 9 presents the time profile of $\delta \mathbf{B}$ measured by $\mathrm{C}_{1}$ (solid line) and $\mathrm{C}_{4}$ (dashed line) for the 2-s periods around $t_{\text {sing }}^{\mathrm{cl}}$ and $t_{\text {sing }}^{\mathrm{c4}}$ in the local minimum variance frame of $\mathrm{C}_{1}$. The small jumps in the $C_{1}$ data $(\sim 4 \mathrm{nT})$ are due to the STAFF search coil (SC) calibration method.

[35] The correlations in equation (3) indicate that it is highly probable that $\mathrm{C}_{1}$ and $\mathrm{C}_{4}$ observe the same structure. Thus its parallel dimension should be greater than the $\mathrm{C}_{1}-$ $\mathrm{C}_{4}$ separation $\delta_{\| 14}=620 \mathrm{~km}$ parallel to $\mathbf{B}_{m s h}$. In Figure 10 we present the projection of the four spacecraft on a plane perpendicular to $\mathbf{B}_{m s h}$; the arrow represents the direction of
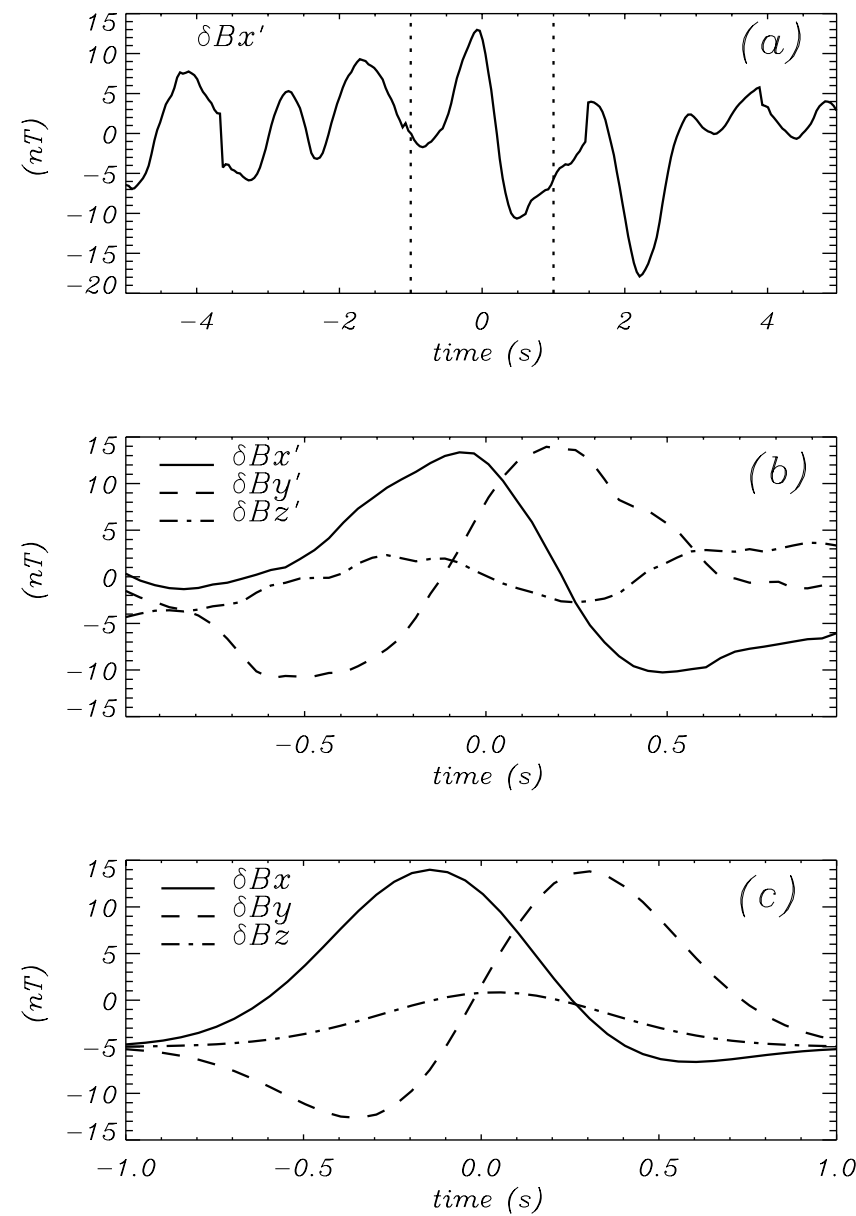

Figure 8. Magnetic field fluctuations, taking $\tau \simeq-420 \mathrm{~s}$ $(1755: 16 \mathrm{UT})$ as the origin of time. (a) Fluctuations $\delta B_{x^{\prime}}$ during $10 \mathrm{~s}$ around $\tau$. (b) Fluctuations of the magnetic field components $\left(\delta B_{x^{\prime}}, \delta B_{y^{\prime}}, \delta B_{z^{\prime}}\right)$ for the 2-s period around $\tau$. (c) The $z$-aligned current tube simulation $\left(\delta B_{x}, \delta B_{y}, \delta B_{z}\right)$. 

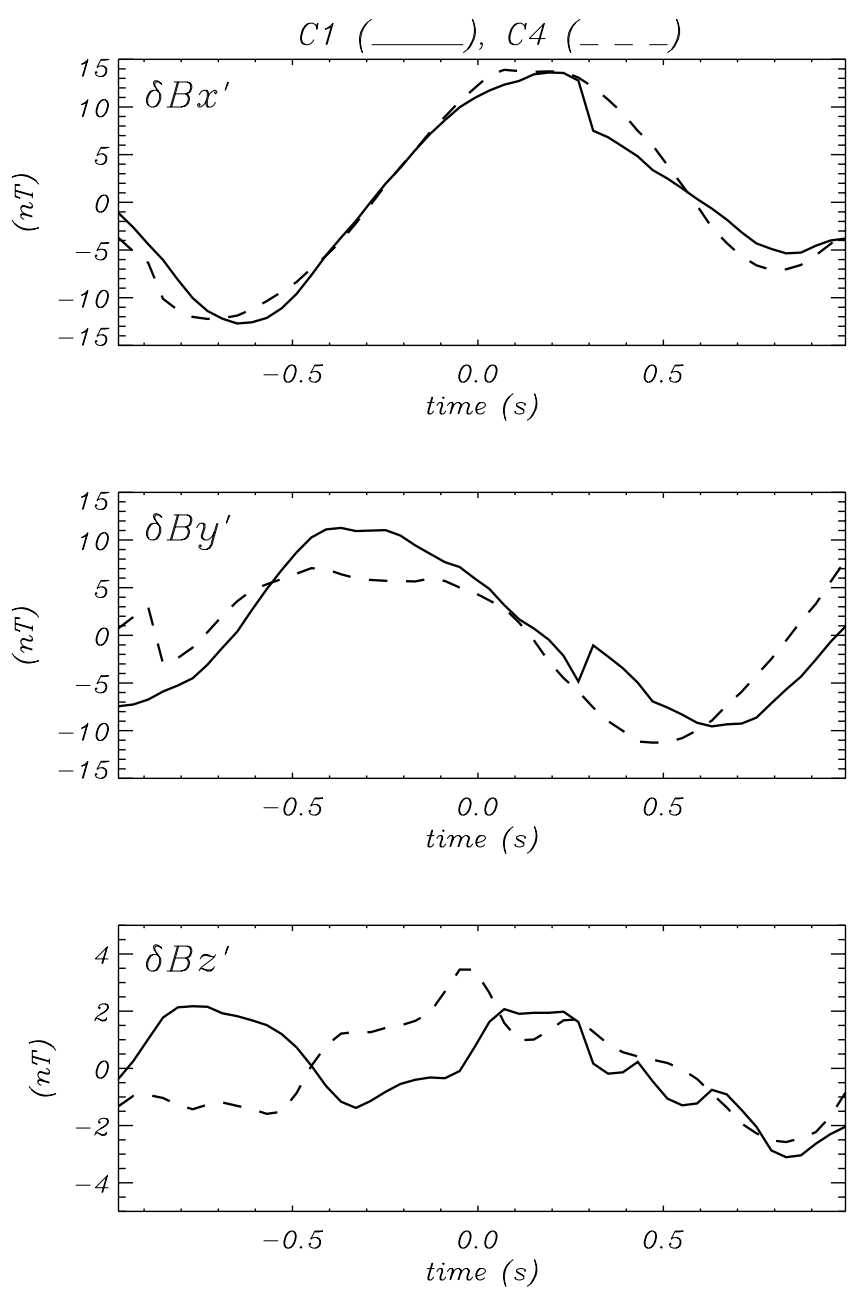

Figure 9. Profile of the magnetic field components measured by $\mathrm{C}_{1}$ around 1755:10 UT (solid line) and by $\mathrm{C}_{4}$ around 1755:11 UT (dashed line).

the plasma bulk velocity in this plane. The time delay $\Delta t_{14} \simeq$ $1.4 \mathrm{~s}$ and the separation $\delta R_{\perp 14} \simeq 200 \mathrm{~km}$ can then be used to determine the displacement velocity of the structure $V_{*} \simeq$ $140 \mathrm{~km} / \mathrm{s}$ in the plane perpendicular to $\mathbf{B}_{m s h}$. This velocity is calculated assuming that the structure is exactly parallel to the ambient magnetic field, but the angle of $10^{\circ}$ between $\mathbf{B}_{m s h}$ and the axis of the structure (an average value over the several structures) can change this result from 70 to $230 \mathrm{~km} / \mathrm{s}$. This last value is very close to the plasma bulk velocity.

[36] A similar profile of magnetic field variation can be obtained with a very simple two-dimensional model, where a cylindrical current tube of radius $R_{0}$ is flowing along an ambient magnetic field $\mathbf{B}=B \mathbf{e}_{z}$. Assuming that all the spatial dependencies are in the $(x, y)$ plane, the model is characterized by two functions, $\delta B_{z}(x, y)$ and the $z$ component $A(x, y)$ of the potential vector $\mathbf{A}=A \mathbf{e}_{z}$ of the magnetic fluctuation, which we take as Gaussian so that

$$
\begin{gathered}
\delta B_{z}=\Delta B_{\|} \exp \left(-R^{2} / R_{0}^{2}\right) \\
A=\left(\frac{\Delta B_{\perp}}{\Delta B_{\|}}\right) R_{0} \delta B_{z},
\end{gathered}
$$

where $\Delta B_{\|}$and $\Delta B_{\perp}$ characterize the parallel and perpendicular amplitude, respectively, of the magnetic fluctuations ( $R$ is the distance to the tube axis, $R^{2}=x^{2}+y^{2}$ ). Since $\delta \mathbf{B}=$ $\delta B_{z} \mathbf{e}_{z}+\nabla \times \mathbf{A}, \delta B_{x}=\left(-2 y / R_{0}^{2}\right) A$ and $\delta B_{y}=\left(2 x / R_{0}^{2}\right) A$. The source of this magnetic perturbation is a field-aligned localized current tube given by the Maxwell equation $\nabla \times$ $\delta \mathbf{B}=\mu_{0} \mathbf{j}$ :

$$
\mathbf{j}=2 \frac{\delta B_{z}}{\mu_{0} R_{0}}\left[-\frac{y}{R_{0}}, \frac{x}{R_{0}}, 2 \frac{\Delta B_{\perp}}{\Delta B_{\|}}\left(1 \frac{R^{2}}{R_{0}^{2}}\right)\right] .
$$

This current has a maximal longitudinal current at the center of the tube and maximal transversal current at the tube border.

[37] An instrument carried by a spacecraft crossing the structure in the $(x, y)$ plane along a trajectory $\rho(\mathrm{t})=\boldsymbol{\rho}_{0}+\mathbf{V}_{*} t$ with a velocity $\mathbf{V}_{*}\left(\rho_{0}\right.$ is the spacecraft position at time $t=$ 0 ) would measure a magnetic field fluctuation $\left\{\delta B_{x}[\rho(t)]\right.$, $\left.\delta B_{y}[\boldsymbol{\rho}(t)], \delta B_{z}[\boldsymbol{\rho}(t)]\right\}$ which is qualitatively similar to the observed one once the parameters are correctly adjusted. In particular, using $R_{0}=60 \mathrm{~km}, \Delta B_{\|} \simeq 15 \mathrm{nT}, \Delta B_{\perp} / \Delta B_{\|} \simeq 2$, and the distance of closest approach to the tube axis $30 \mathrm{~km}$, we obtain the profiles of $\delta B_{x}, \delta B_{y}$, and $\delta B_{z}$ shown in Figure $8 \mathrm{c}$. This model is oversimplified and does not rely on a firm physical basis. It can be used nevertheless for the purpose of illustration and dimensional analysis.

[38] Thus one may conclude that during the time interval from 500 to $350 \mathrm{~s}$ before the shock crossing, on the 2$\mathrm{s}$ timescale, satellites $\mathrm{C}_{1}$ and $\mathrm{C}_{4}$ observe field-aligned current tubes with a perpendicular cross section of the order of $60 \mathrm{~km}$ and a parallel size $>600 \mathrm{~km}$. Satellites $\mathrm{C}_{2}$ and $\mathrm{C}_{3}$ also observe similar structures on the 2-s timescale in this region. The space separations in the plane perpendicular to the magnetic field $\delta R_{\perp 12} \simeq 650 \mathrm{~km}$ and $\delta R_{\perp 24} \simeq$ $720 \mathrm{~km}$ are much larger than $R_{0}$ estimated from the model (equation (4)); therefore we cannot be sure that $\mathrm{C}_{2}$ observes the same structures as $\mathrm{C}_{1}$ and $\mathrm{C}_{4}$. The separation $\delta R_{\perp 13} \simeq$

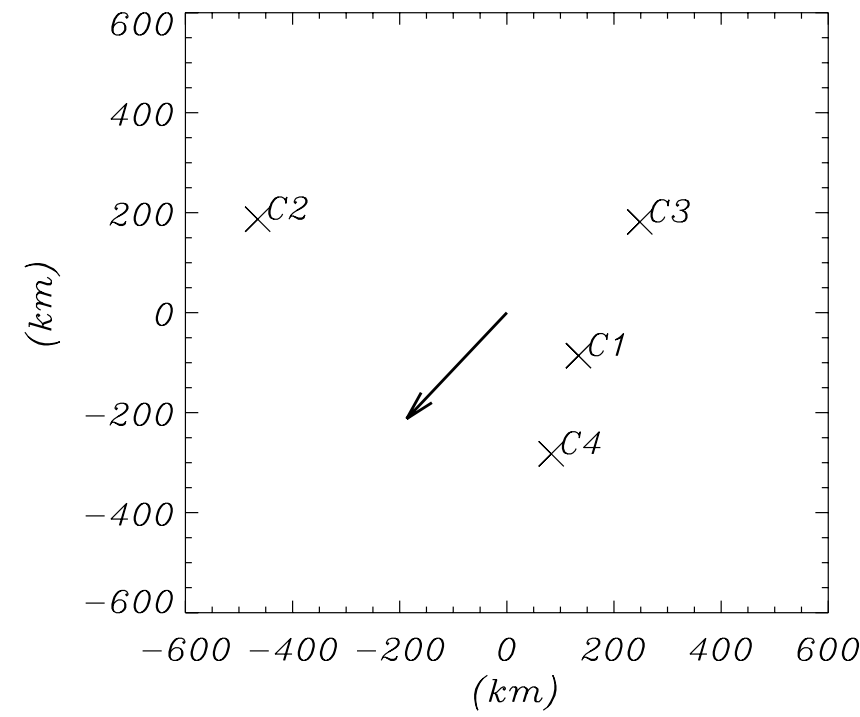

Figure 10. Relative positions of $\mathrm{C}_{1}, \mathrm{C}_{2}, \mathrm{C}_{3}$, and $\mathrm{C}_{4}$ for the studied shock crossing projected on the plane perpendicular to $\mathbf{B}_{m s h}$. The arrow represents the direction of the bulk velocity in this plane. 



Figure 11. Schematic presentation of the processes sequence discussed in text: generation and filamentation of AIC waves in the downstream region of the perpendicular bow shock.

$290 \mathrm{~km}$ is not much larger than $\delta R_{\perp 14}$, but during $20 \mathrm{~s}$ before $\tau$, there are no data on $\mathrm{C}_{3}$, so we cannot properly compare structures observed by $\mathrm{C}_{3}$ with those discussed in this section. More detailed comparisons between the four satellites will be the subject of a future work. We can conclude that just downstream of the region where coherent Alfvén ion cyclotron waves are observed, one finds a region more or less loosely filled with current tubes parallel to the local ambient magnetic field.

\section{Discussion and Conclusion}

[39] It is well known, both theoretically and observationally, that AIC waves may be destabilized by proton and $\alpha$ particle pressure anisotropies downstream of a quasiperpendicular, supercritical bow shock [Anderson et al., 1994; Gary et al., 1994]. This is the case for the shock crossing studied here, which falls in a regime of temperature anisotropies and $\beta_{p}$ where Alfvén ion cyclotron waves are unstable. As predicted by previous authors, we observed two AIC modes propagating approximately along the downstream average magnetic field and corresponding to the destabilizing effects of the $\alpha$ and proton temperature anisotropies, in agreement with the linear theory of the instability. Note that the identification of the waves observed by one satellite was based on the polarization study and on the wave vector direction obtained by the minimum variance method. With the help of the four Cluster satellites the determination of the wave dispersion is possible. To our knowledge, an accurate AIC wave identification in the downstream region of a quasiperpendicular shock is made here for the first time.

[40] Farther downstream in the magnetosheath but still close to the shock front, the AIC waves disappear. Simultaneously, the pressure anisotropies decrease, and largeamplitude, localized fluctuations appear at timescales and spatial scales smaller than the period and wavelength of the AIC waves. These fluctuations may be interpreted as fieldaligned current tubes and last until the previous shock crossing is reached at 1738:22 UT. No quasi-monochromatic AIC waves are observed downstream of this shock, the Cluster spacecraft finding themselves immediately in a region with current tubes similar to those described below.
[41] Thus, in the downstream region of a perpendicular bow shock, two different phenomena are observed successively: coherent, plane AIC waves propagating along $\mathbf{B}_{m s h}$, followed by a region where the magnetic fluctuations take the form of current tubes. Even if the geometry and the length scales of the two phenomena are very different, they are observed in adjacent regions. This is the most important result of this work.

[42] A possible interpretation for the observed symmetry breaking is the filamentation instability [Laveder et al., 2002] of the quasi-monochromatic AIC waves observed downstream of the shock. This instability takes place in a plasma with $\beta \geq 1$ and leads to the collapse of the finite amplitude dispersive Alfvén wave into intense field-aligned magnetic filaments with a cross section of the order of $c / \omega_{p i}$. This instability has been extensively studied for parallelpropagating waves in Hall-MHD without kinetic effects. In our case the conditions are favorable for the filamentation instability. In particular, the total plasma $\beta$ in the magnetosheath exceed the unity: $\left(\beta_{p}+\beta_{e}\right)_{m s h} \simeq 1.1$. Furthermore, the AIC characteristics, such as the frequency, wavelength, and polarization and their direction of propagation, more or less along the ambient magnetic field, are the correct ones for the development of the instability. The cross section of the current tubes is of the same order as the cross section of the filament seen in the simulations [Laveder et al., 2002]. The structure of this filament is rather complex: An azimuthal magnetic field does not make a complete twist, an axial component has a shear inside the filament, and an electric current within the filament has the same structure (D. Laveder, private communication, 2003). The model we have used in this paper is highly schematic, and a detailed study of the fine structure of the observed filaments will be the subject of a forthcoming paper.

[43] The sequence of processes, discussed in this paper, is summarized in the cartoon of Figure 11: The supercritical quasi-perpendicular bow shock is the source of the strong downstream temperature anisotropy which excites the AIC waves; the waves give rise to the current tubes via the filamentation instability. As mentioned in section 3, the pressure anisotropy remains relatively high in the AIC region and decreases in the tube region, indicating that the filamentation instability plays an important role in the final plasma relaxation in the downstream region.

[44] For the other shock crossings observed that day, the solar wind and shock parameters were roughly in the same range; it is therefore surprising that the combination of AIC waves and current tubes was found for only one other shock crossing, occurring at 1717:49 UT. However, for all of them we were able to identify field-aligned current tubes downstream but close to the shock front. This suggests that the filamentation instability is so fast that AIC waves cannot be observed if the geometry of the crossing is not favorable. Detailed comparisons with theoretical models of this nonlinear instability, including kinetic effects, are necessary to support these conclusions. This will be the subject of further studies.

\section{Appendix A}

\section{A1. Wavelet Transformation: Basic Concepts}

[45] In many cases of interest one has to analyze a signal $S(t)$ whose spectral content varies with time. The methods 

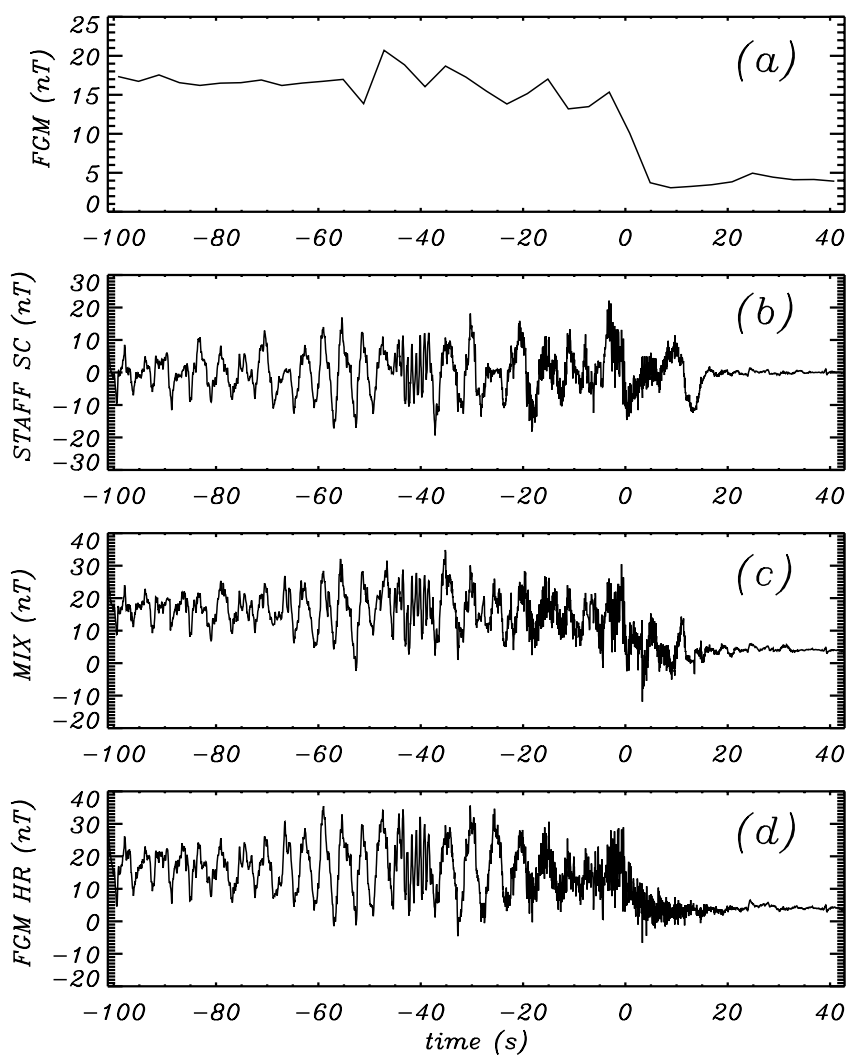

Figure A1. Data merging for $B_{X}(\mathrm{nT})$ : (a) FGM data $\left[B_{X}(t)\right]_{F}$, (b) Cluster Spatio-Temporal Analysis of Field Fluctuations (STAFF) search coil waveforms $\left[B_{X}(t)\right]_{S}$, (c) mixed data $\left[B_{X}(t)\right]_{\text {mix }}$, and (d) FGM high resolution.

which are commonly used to study this variation of the spectral content (within the limits of the uncertainty principle, which states that one cannot obtain arbitrary good localization simultaneously in time and frequency, $\Delta f \Delta t \geq$ $\mathrm{C}^{\text {te }}$ ) are the windowed Fourier transform (WFT) and the wavelet transform (WT).

[46] In the WFT, time localization is achieved by using a windowing function $g(t)$ and then taking the Fourier transform

$$
G S(f, t)=\int_{-\infty}^{+\infty} S\left(t^{\prime}\right) g\left(t^{\prime}-t\right) e^{-i 2 \pi f t^{\prime}} d t^{\prime}
$$

where $g(t)$ is a real function which vanishes for $t \geq T$. This transform is energy preserving and invertible. It is characterized by the fact that the analyzing kernel $g\left(t^{\prime}-\right.$ t) $e^{-i 2 \pi f t^{\prime}}$ has the same support for all $f$ and $t$, while the number of cycles varies with $f$, with the consequence that frequency resolution is $\Delta f \simeq 1 / T$ and the time localization $\Delta t \simeq T$ does not depend on the frequency.

[47] In the WT one uses an analyzing function which has a narrow support for high frequencies and a large one for low frequencies. This is achieved by using a twoparameter family of functions called wavelets, $\psi_{f, t}\left(t^{\prime}\right) \equiv$ $\sqrt{f} \psi\left[f\left(t^{\prime}-t\right)\right]$, derived from a "mother" wavelet $\psi(t)$, which has a finite support around $t=0$. One of the parameters is the translation parameter $t$, as in the win- dowed Fourier transform case; the other parameter is a dilation (or scale) parameter $f>0$ corresponding to a local frequency, $1 / f$ being the temporal scale. Making the convolution of the signal $S(t)$ with a member of the family of functions $\psi_{f, t}\left(t^{\prime}\right)$, we obtain the corresponding wavelet coefficient

$$
W S(f, t)=\int_{-\infty}^{+\infty} S\left(t^{\prime}\right) \sqrt{f} \psi\left[f\left(t^{\prime}-t\right)\right] d t^{\prime}
$$

[48] Changing the value of $f$ has the effect of dilating $(1 / f>1)$ or contracting $(1 / f<1)$ the analyzing function $\psi_{f, t}\left(t^{\prime}\right)$, while changing $t$ has the effect of translating the support of this function toward the point $t$. In the wavelet transform the number of cycles in the wavelet $\psi_{f, t}\left(t^{\prime}\right)$ does not change with the scale parameter $1 / f$, but the size of its support does. It is small for small $1 / f$, so the wavelet transform picks up higher-frequency components and vice versa. The choice of the function $\psi(t)$ is not unique, but $\psi(t)$ must have a finite energy, a sufficiently fast decay for large values of $|t|$ and zero mean.

[49] The signals we have to deal with are discrete ones, with a sampling time $t_{s}$. A complete orthogonal wavelet basis may then be constructed if a logarithmic uniform discretization of spacing and scales is chosen, for example,

$$
t_{n}=n 2^{m} t_{s}, \quad f_{m}=\frac{1}{2^{m} t_{s}}, \quad m \geq 1 .
$$

At scale $m$ the time resolution is $2^{m} t_{s}=\Delta t_{m}$.

[50] In this work we have used two wavelet transforms, depending on whether we need a good time resolution or a good frequency resolution [see, e.g., Kumar and Foufoula-Georgiou, 1994]. The first one is the Haar wavelet (HW),

$$
\psi(t)=\left\{\begin{array}{cc}
1, & 0<t \leq 1 / 2 \\
-1, & 1 / 2<t \leq 1 \\
0, & \text { elsewhere. }
\end{array}\right.
$$

Note that computing the Haar coefficients of a sampled signal $S\left(t_{i}\right)$ at a given scale $m$ is equivalent to taking the difference between the average of the signal over two consecutive intervals of length $2^{m}$ points. At the highest scale this procedure is equivalent to taking the difference between two neighboring points of the signal, which provides the highest time resolution but the worst frequency resolution of the transformation. Therefore the HW transform is convenient for detecting fast and local variations, which we call the "singular points." The second WT is the Morlet wavelet

$$
\psi(t)=\pi^{-1 / 4} e^{-(i 2 \pi f t)} e^{-t^{2} / 2},
$$

where $(2 \pi f) \geq 5$, which represents a good compromise between time and scale resolution; it is well suited 
to detecting frequency peaks in an inhomogeneous environment.

\section{A2. Data Merging}

[51] The search coil (SC) magnetometer of the STAFF SC experiment measures magnetic waveforms with a sampling time of $t_{s}=0.04 \mathrm{~s}$ in the normal bit rate mode. Frequencies smaller than the spacecraft spin rate $\left(1 / t_{R}, t_{R} \simeq 4 \mathrm{~s}\right)$ are filtered out so that the useful signal covers the $0.25-$ $12.5 \mathrm{~Hz}$ frequency range. In many cases, however, it is convenient to have magnetic waveforms which also contain information about the large-scale variations, information which may be found in the FGM data, averaged, and sampled at $4 \mathrm{~s}$. We are therefore led to merge the STAFF SC signal with the FGM signal, which can be done in many ways.

[52] Here we use the Haar wavelet transform (the WFT could be used as well, the main requirement being that the transform is invertible). To illustrate the procedure, consider the $X$ th component of the magnetic field $\left[B_{X}(t)\right]_{F}$ measured by FGM (see Figure A1a) and $\left[B_{X}(t)\right]_{S}$ measured by STAFF SC (see Figure A1b). We begin by interpolating the $\left[B_{X}(t)\right]_{F}$ signal to the STAFF measurement times; then we apply the Haar wavelet transform to both the STAFF waveforms and the interpolated FGM waveforms, obtaining the wavelet coefficients $\left[W B_{X}(m, t)\right]_{S}$ and $\left[W B_{X}(m, t)\right]_{F}$, respectively. The period of rotation lies in the $m=m_{*}=6$ scale since $2^{m *}<t_{R}<2^{m *+1}$. We then combine these two sets of wavelet coefficients to obtain the wavelet coefficients $\left[W B_{X}(m, t)\right]_{\text {mix }}$ of a mixed signal, equal to $\left[W B_{X}(m, t)\right]_{F}$ for scales larger than $m_{*}$ and to $\left[W B_{X}(m, t)\right]_{S}$ for scales smaller than $m_{*}$ :

$$
\left[W B_{X}(m, t)\right]_{\text {mix }}=a_{m}\left[W B_{X}(m, t)\right]_{F}+b_{m}\left[W B_{X}(m, t)\right]_{S},
$$

where $a_{m}$ and $b_{m}$ are weight functions

$$
\begin{gathered}
a_{m}=\left\{\begin{array}{cc}
1, & m \geq m_{*} \\
2^{-2(m-5)}, & m<m_{*}
\end{array}\right. \\
b_{m}=1-a_{m} .
\end{gathered}
$$

We now apply the inverse wavelet transformation using the set of $\left[W B_{X}(f, t)\right]_{\text {mix }}$ to get the final "mixed" signal $\left[B_{X}(t)\right]_{\text {mix }}$ (see Figure A1c). In Figure A1d we also present the FGM high-resolution signal to compare with our resulting signal. One can see that the signals are very similar, with the fluctuation amplitude being slightly higher for the high-resolution FGM data than for the merged signal; a more detailed comparison of these two signals will be made in a future work.

[53] Acknowledgments. We thank the ACE SWEPAM instrument team and the ACE Science Center for providing the ACE data.

[54] Shadia Rifai Habbal thanks Dimitri Laveder and another referee for their assistance in evaluating this paper.

\section{References}

Anderson, B. J., S. A. Fuselier, S. P. Gary, and R. E. Denton (1994), Magnetic spectral signatures in the Earth's magnetosheath and plasma depletion layer, J. Geophys. Res., 99, 5877-5891.

Balikhin, M. A., T. Dudok de Wit, V. Krasnosel'skikh, W. A. C. MierJedrzejeowicz, and W. Baumjohann (1997), Experimental determination of the dispersion of waves observed upstream of quasi-perpendicular shock, Geophys. Res. Lett., 24, 787-790.

Balikhin, M. A., I. Bates, and M. Dunlop (2000), Minimum variance free identification of the composition of space plasma turbulence and nonlinear processes in it, in Proceeding of the Cluster-II Workshop on Multiscale/Multipoint Plasma Measurements, edited by R. A. Harris, Eur. Space Agency Spec. Publ., ESA SP-449, 287-290.

Balogh, A., et al. (2001), The Cluster magnetic field investigation: Overview of in-flight performance and initial results, Ann. Geophys., 19, $1207-1217$.

Cornilleau-Wehrlin, N., et al. (1997), The Cluster Spatio-Temporal Analysis of Field Fluctuations (STAFF) experiment, Space Sci. Rev., 79, 107-136.

Cornilleau-Wehrlin, N., et al. (2003), First results obtained by the Cluster STAFF experiment, Ann. Geophys., 21, 437-456.

Décréau, P. M. E., et al. (1997), WHISPER, A resonance sounder and wave analyser: Perfomances and perspectives for the Cluster mission, Space Sci. Rev., 79, 157-193.

Fuselier, S. A., D. M. Klumpar, E. G. Shelley, B. J. Anderson, and A. J. Coates (1991), $\mathrm{He}^{2+}$ and $\mathrm{He}^{+}$dynamics in the subsolar magnetosheath and plasma depletion layer, J. Geophys. Res., 96, 21,095-21,104.

Gary, S. P., P. D. Convery, R. E. Denton, S. A. Fuselier, and B. J. Anderson (1994), Proton and helium cyclotron anisotropy instability thresholds in the magnetosheath, J. Geophys. Res., 99, 5915-5921.

Johnstone, A. D., et al. (1997), PEACE: A Plasma Electron and Current Experiment, Space Sci. Rev., 79, 351-398.

Kumar, P. and E. Foufoula-Georgiou (1994), Wavelet analysis and its applications: An introduction, in Wavelets in Geophysics, edited by E. Foufoula-Georgiou and P. Kumar, pp. 1-43, Academic, San Diego, Calif.

Laveder, D., T. Passot, and P. L. Sulem (2002), Transverse dynamics of dispersive Alfvén waves: Direct numerical evidence of filamentation, Phys. Plasmas, 9, 293-304.

Leroy, M. M., D. Winske, C. C. Goodrich, C. S. Wu, and K. Papadopoulos (1982), The structure of perpendicular bowshocks, J. Geophys. Res., 87, $5081-5094$.

Maksimovic, M., S. D. Bale, T. S. Horbury, and M. Andre (2003), Bow shock motions observed with CLUSTER, Geophys. Res. Lett., 30(7), 1393, doi:10.1029/2002GL016761.

McComas, D. J., S. J. Bame, P. Barker, W. C. Feldman, J. L. Phillips, P. Riley, and J. W. Griffee (1998), Solar Wind Electron Proton Alpha Monitor (SWEPAM) for the Advanced Composition Explorer, Space Sci. Rev., 86, 563-612.

Paschmann, G., N. Sckopke, S. J. Bame, and J. T. Gosling (1982), Observations of gyrating ions in the foot of a nearly perpendicular bowshock, Geophys. Res. Lett., 9, 881.

Rème, H., et al. (1997), The Cluster Ion Spectrometry (CIS) experiment, Space Sci. Rev., 79, 303-350.

Rezeau, L., A. Roux, and C. T. Russell (1993), Characterization of smallscale structures at the magnetopause from ISEE measurements, J. Geophys. Res., 98, 179-186.

Rönnmark, K. (1982), Waves in homogeneous, anisotropic, multicomponent plasmas, Kiruna Geophys. Inst. Rep. 179, 56 pp., Swed. Inst. of Space Phys., Univ. of Umeà, Umeà, Sweden.

Rönnmark, K. (1983), Computation of the dielectric tensor of a Maxwellian plasma, Plasma Phys., 25, 699-701.

Sahraoui, F., et al. (2003), ULF wave identification in the magnetosheath: K-filtering technique applied to Cluster II data, J. Geophys. Res., 108(A9), 1335, doi:10.1029/2002JA009587.

Schwartz, S. J. (1998), Shock and discontinuity normals, Mach numbers, and related parameters, in Analysis Methods for Multi-Spacecraft Data, edited by G. Paschman and P. W. Daly, ISSI Sci. Rep. SR-001, pp. 249270, Eur. Space Agency, Paris.

Schwartz, S. J., D. Burgess, and J. J. Moses (1996), Low-frequency waves in the Earth's magnetosheath: Present status, Ann. Geophys., 14, 11341150.

Sonnerup, B. U. Ö., and M. Scheible (1998), Minimum and maximum variance analysis, in Analysis Methods for Multi-Spacecraft Data, edited by G. Paschman and P. W. Daly, ISSI Sci. Rep. SR-001, pp. 185-220, Eur. Space Agency, Paris.

Yoon, P. H. (1992), Quasi-linear evolution of Alfven-ion-cyclotron and mirror instabilities driven by ion temperature anisotropy, Phys. Fluids $B, 4,3627-3637$.

Zhao, X., K. W. Ogilvie, and Y. C. Whang (1991), Modeling the effects of fast shocks on solar wind minor ions, J. Geophys. Res., 96, 5437-5445.

O. Alexandrova, C. Lacombe, M. Maksimovic, and A. Mangeney, LESIA/CNRS, Observatoire de Paris, 5 pl. Jules Janssen, 92195 Meudon, France. (olga.alexandrova@obspm.fr; catherine.lacombe@obspm.fr; milan. maksimovic@obspm.fr; mangeney@despace.obspm.fr) 
J.-M. Bosqued, CESR, 9, av. du Colonel Roche, F-31028 Toulouse, France. (jean-michel.bosqued@cesr.fr)

N. Cornilleau-Wehrlin, CETP, 10-12, av. de l'Europe, F-78140 Vélizy, France. (nicole.cornilleau@cetp.ipsl.fr)

P. M. E. Décréau, LPCE/CNRS, 3A, av. de la recherche scientifique, F-45071 Orleans, France. (pdecreau@cnrs-orleans.fr)
A. N. Fazakerley, Mullard Space Science Laboratory, Holmbury, St. Mary, Dorking, Surrey RH5 6NT, UK. (anf@mssl.ucl.ac.uk)

E. A. Lucek, Space and Atmospheric Physics, Imperial College, Prince Consort Road, London SW7 2BZ, UK. (e.lucek@imperial.ac.uk)

P. Travnicek, Institute of Atmospheric Physics, Prague 14131, Czech Republic. (trav@alenka.ufa.cas.cz) 